\section{OP0319 LOW AND MODERATE PHYSICAL ACTIVITY REDUCES LOCALISED IL-1B IN AN ACUTE MOUSE MODEL OF GOUT BY DOWN-REGULATING TLR2 EXPRESSION ON CIRCULATING NEUTROPHILS}

K. Jablonski ${ }^{1 *}$, B. Sandoval' ${ }^{1}$, P. Harb ${ }^{1}$, A. Kalyanasundaram ${ }^{2}$, J.M. Hampton ${ }^{1}$, W. N. Jarjour ${ }^{1}$, N. Schlesinger ${ }^{3}$, N.A. Young ${ }^{1}{ }^{1}{ }^{1}$ Rheumatology and Immunology; ${ }^{2}$ Physiology and Cell Biology, The Ohio State University Wexner Medical Center, Columbus; ${ }^{3}$ Rheumatology, Rutgers Robert Wood Johnson Medical School, New Brunswick, USA

Background: While physical activity was originally believed to exacerbate inflammation in rheumatic disease, recent studies have shown significant reductions in inflammation with regular exercise. It has been previously shown that down-regulation of toll-like receptor (TLR)2 and TLR4 expression correlates with increased physical activity in humans. Furthermore, both TLR2 and TLR4 knockout mice are resistant to monosodium urate (MSU) crystal-induced gout. Additionally, mesenchymal stem cells (MSCs) can be immunosuppressive by secreting IL-1 receptor antagonist (IL-1RA) and have also been shown to be up-regulated with exercise. Objectives: The aim of this study was to investigate the mechanism by which exercise suppresses gouty inflammation and to define the potential roles of TLR2, TLR4, and MSCs in the process.

Methods: NFאB reporter mice [BALB/C-Tg(NFKB-RE-luc)-Xen] were exercised daily by treadmill walking ( $45 \mathrm{~min} /$ day for 2 weeks) at low intensity $\left(35 \% \mathrm{VO}_{2}\right.$ max; $8 \mathrm{~m} / \mathrm{min})$, moderate intensity $\left(55 \% \mathrm{VO}_{2} \mathrm{max} ; 11 \mathrm{~m} / \mathrm{min}\right)$, and high intensity $(75 \%$ $\mathrm{VO}_{2} \max ; 15 \mathrm{~m} / \mathrm{min}$ ). Mice were then injected with MSU crystals $(0.5 \mathrm{mg})$ into the tibio-tarsal joint (ankle). Localised NFKB activity was measured 16 hours later in the injected ankle by bioluminescent imaging. Tissue was collected and processed for immunohistochemical $(\mathrm{IHC})$ analysis and whole blood was collected for both flow cytometry and serum analysis.

Results: Mice in the low/moderate intensity exercise groups had decreased inflammation, $\mathrm{F} 4 / 80^{+}$macrophages, and $\mathrm{MPO}^{+}$neutrophils at the site of MSU injection compared to high intensity and non-exercised controls. Similarly, bioluminescent imaging of NFKB activity was significantly reduced locally in both low/ moderate intensity groups compared to high-intensity and non-exercised controls. Surface expression of TLR4 on peripheral monocytes or neutrophils showed little difference by flow cytometry, while TLR2 expression on peripheral neutrophils was significantly reduced. In concordance, localised IL-1 $\beta$ expression via IHC was reduced in low/moderate intensity exercise conditions. IL-1RA expression correlated with IL-1 $1 \beta$ induction locally by $\mathrm{IHC}$ and was elevated in serum. Also, bone marrow-derived MSCs were significantly reduced in low/moderate intensity exercise compared to high-intensity or non-exercised controls.

\section{TLR2+ Neutrophils in peripheral blood}
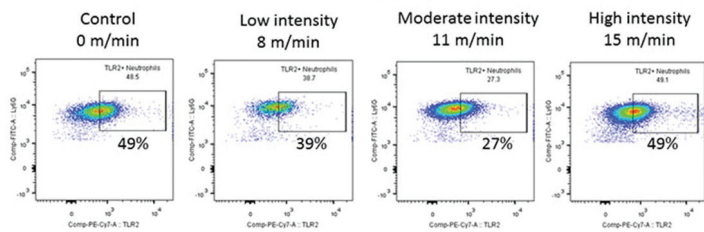

IL-1 $\beta$ in joint space
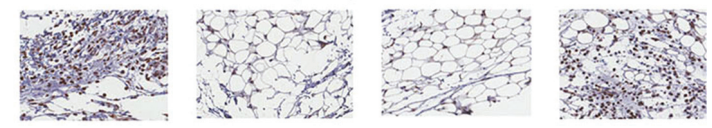

Conclusions: These data show that while low/moderate intensity exercise regimens can reduce the localised MSU crystal-induced inflammation, high intensity training negates this response. Moreover, the exercise-mediated suppression of $\mathrm{NF} \kappa \mathrm{B}$ activity and IL-1 $\beta$ expression locally can be partially explained by a reduction in peripheral neutrophil recruitment via downregulation of TLR2 expression in the peripheral blood. Although not clearly defined mechanistically in this study, our results also suggest that MSCs may contribute to this immunosuppressive response and are mobilised out of the bone marrow with low/moderate intensity exercise.

\section{REFERENCES:}

[1] Khanna D, et al. Arthritis Care Res 2012;64:1447.

[2] Agudelo CA, et al. Arthritis Rheum 1972;15:609.

[3] Liu-Bryan R, et al. Arthritis Rheum 2005;52:2936.

Acknowledgements: Support provided by Ironwood Pharmaceuticals, Cambridge MA 02142

Disclosure of Interest: None declared

DOI: 10.1136/annrheumdis-2018-eular.4764

\section{OP0320 \\ S100A9 HAMPERS OSTEOCLAST DIFFERENTIATION FROM CIRCULATING PRECURSORS BY REDUCING THE EXPRESSION OF RANK}

M.H. Van Den Bosch ${ }^{1}$, I. Di Ceglie ${ }^{1}$, T. Vogl| ${ }^{2}$, J. Roth ${ }^{2}$, C.S. Goodyear ${ }^{3}$, P.M. van der Kraan ${ }^{1}$, A.B. Blom ${ }^{1}$, P.L. van Lent ${ }^{1} .{ }^{1}$ Experimental Rheumatology, Radboud university medical center, Nijmegen, Netherlands; ${ }^{2}$ Institut für Immonologie, Münster, Germany; ${ }^{3}$ Institute of Infection, Immunity and Inflammation, Glasgow, UK

Background: High levels of the damage-associated molecular patterns (DAMPs) S100A8 and S100A9 are produced in the synovium during both experimental and human rheumatoid arthritis (RA). These alarmins have been implicated in inflammation-induced bone resorption. We and others have previously shown that stimulation of mature osteoclasts with S100A8/A9 results in increased numbers and bone resorptive activity. In agreement, reduced bone destruction was observed after induction of experimental RA models in S100a9 $9^{-/}$mice. However, the effects of S100A8/A9 on monocyte-to-osteoclast differentiation remain elusive.

Objectives: Here, we investigated the effects of S100A9 on CD14 ${ }^{+}$monocytes and their potential to differentiate into osteoclasts.

Methods: CD14 ${ }^{+}$monocytes were isolated from buffy coats of healthy donors using density gradient centrifugation and magnetic cell sorting. Cells were differentiated into osteoclasts with macrophage colony-stimulating factor (M-CSF) and Receptor activator of nuclear factor kappa-B (RANK) ligand (RANKL) in the presence or absence of S100A9. mRNA expression was determined by RT-qPCR and protein expression was determined using Luminex analysis. Moreover, osteoclast differentiation was assessed using Tartrate-resistant acid phosphatase (TRAP) staining and the resorptive capacity was determined using mineral-coated plates RANK protein expression was assessed using FACS.

Results: We observed that S100A9 stimulation of monocytes resulted in a strong induction of various pro-inflammatory factors, such as interleukin (IL) 1及, IL6, IL8, and tumour necrosis factor (TNF) $\alpha$ after 24 hour, both on the mRNA and protein level. Interestingly, we observed a strong decrease in the number of multinucleated osteoclasts as determined by TRAP staining, at day 6 and 8 after start of the cultures. In agreement with this, the cells showed a strongly reduced resorptive capacity after 10 days of culture. We demonstrated that already a 24 hour stimulation with S100A9 strongly reduced the osteoclastogenic potential of the $\mathrm{CD}_{14}{ }^{+}$monocytes. Finally, to determine the mechanism of how this short S100A9 stimulation might reduce the osteoclast development, we determined the protein expression of the RANK receptor, which is crucial for osteoclast differentiation. We observed that S100A9 stimulation hampered the M-CSF-induced upregulation of RANK after 24 hours, suggesting that this underlies the hampered osteoclast differentiation. Interestingly, this S100A9-induced decreased RANK expression could be reversed by addition of the TNF $\alpha$-inhibitor etanercept, but not by addition of IL1 receptor antagonist.

Conclusions: Whereas S100A8/A9 have been previously shown to stimulate the numbers and resorptive capacity of mature osteoclasts, we here show that stimulation of monocytes with S100A9 strongly inhibits their osteoclastogenic potential, possibly via TNF $\alpha$-induced reduction of RANK expression. This suggests that S100A8/A9 does not solely stimulate osteoclast formation and function but rather that the timing of exposure to S100A8/A9 is an important determinant for monocyte-to-osteoclast differentiation.

Disclosure of Interest: None declared

DOI: 10.1136/annrheumdis-2018-eular.3689

FRIDAY, 15 JUNE 2018

\section{Pathophysiology and biomarkers in PsA: what impact?}

\section{OP0321 PRECISION MEDICINE USING DIFFERENT BIOLOGICAL DMARDS BASED ON CHARACTERISTIC PHENOTYPES OF PERIPHERAL T HELPER CELLS IN PSORIATIC} ARTHRITIS

1. Miyagawa, S. Nakayamada, K. Nakano, S. Kubo, S. Iwata, Y. Miyazaki, M. Yoshikawa, H. Yoshinari, Y. Tanaka. The First Department of Internal Medicine, University of Occupational and Environmental Health, Japan, Kitakyusyu, Japan

Background: Biological DMARDs targeting TNF- $\alpha$, IL-17, and IL-12/23 (p40) are available. The high efficacy of these drugs has been proven in numerous clinical trials. However, there are some cases in which a change from one bDMARDs to another one is necessary because of the refractory nature of the disease, and there is no established method to select the optimal bDMARDs according to the individual case, despite the fact that various drugs are available.

Objectives: We sought to investigate the selection of specific biological DMARDs based on characteristic lymphocyte phenotypes for treating PsA.

Methods: we performed this study to evaluate the efficacy of biologics therapy in 64 patients with PsA after 6 months of therapy, and to compare the results of 
peripheral lymphocyte phenotyping using 8-colour flow cytometry, with specific focus on helper T cell subsets, between 26 patients with PSA and healthy donors. In addition, the therapeutic response of 26 patients in whom the optimal bDMARDs was strategically chosen based on the results of peripheral lymphocyte analysis was evaluated at 6 months of treatment intervention in comparison with 38 patients in whom the standard biological product was used based on the 2011 and 2015 EULAR recommendations. Thus, the possibility of the optimisation of drug selection for bDMARDs therapy based on peripheral blood lymphocyte phenotyping was investigated.

Results: The 26 patients with PsA in the strategic treatment group were classified into the following 4 types based on the peripheral blood analysis: a $\mathrm{CXCR}^{+}{ }^{+} \mathrm{CR} 6{ }^{-}{ }^{-} \mathrm{CD} 38^{+} \mathrm{HLA}-\mathrm{DR}^{+}$activated Th1 cell-predominant type, $\mathrm{CXCR}^{-} \mathrm{CCR}^{+}{ }^{+} \mathrm{CD}^{2} 8^{+} \mathrm{HLA}^{-\mathrm{DR}^{+}}$activated Th17 cell-predominant type, Th1/ Th17-high type, and Th1/Th17-low type. Accordingly, ustekinumab was administered to the activated Th1 cell-predominant patients, secukinumab to the activated Th17 cell-predominant patients, secukinumab or TNF inhibitor to the Th1/Th17high patients, and TNF inhibitor to the Th1/Th17-low patients. At 6 months of strategic treatment, there was a significant decrease in SDAI (from 16.2 to 3.52), DAS28 (ESR) (from 4.13 to 2.27), and PASI (from 8.36 to 2.40). There were no statistically significant differences in background factors at baseline between these 2 groups. Moreover, the proportion of patients with the combined use of MTX was significantly lower in the strategic bDMARDs treatment group. There were significant decreases in TJC, SJC, PGA, CRP, ESR, DAS28 (ESR), SDAI, and PASI in both groups at 6 months of therapy. There were no significant differences in the amounts of these decreases between the two groups. However, at 6 months of therapy, the rate of low disease activity achievement according to SDAI, DAS28 (ESR), and ACR20 response rate was significantly higher in the strategic bDMARDs treatment group.

Conclusions: Strategic treatment in which different bDMARDs were selected according to phenotypic differences in helper $\mathrm{T}$ cells showed significantly higher efficacy than standard bDMARD therapy. The results of this study provide an important guide to the implementation of more effective therapeutic intervention.

Disclosure of Interest: None declared

DOI: 10.1136/annrheumdis-2018-eular.3829

FRIDAY, 15 JUNE 2018

The art of diagnosis of axial SpA

\section{OP0322 ARE WE TREATING WITH BIOLOGICAL THERAPIES WOMEN PATIENTS WITH REAL NON-RADIOGRAPHIC AXIAL SPONDYLOARTHRITIS?}

$\underline{\text { R.E. Nieto }}^{1}$, C. Plasencia ${ }^{2}$, D. Peiteado ${ }^{2}$, A. Villalba $^{2}$, A. Balsa ${ }^{2}$, V. NavarroCompán ${ }^{2}{ }^{1}$ Hospital Provincial de Rosario, Rosario, Argentina; ${ }^{2}$ University Hospital La Paz, IdiPaz, Madrid, Spain

Background: As a result of the development of the ASAS criteria for axial spondyloarthritis (axSpA), a new entity (non-radiographic axSpA -nr-axSpA) was created. In some countries major concerns have been raised with regard to this entity because this could imply administrating TNF inhibitors (TNFi) to non-SpA patients. Especially the possibility of treating women with fibromyalgia has been mentioned.

Objectives: To evaluate if the gender distribution and the pattern of patients have changed in clinical practice since TNFi were approved for nr-axSpA.

Methods: Dataset from a prospective cohort including all patients with axSpA treated with biological therapy (BT) since 2000 till August-2017 in a tertiary hospital was analysed. Patients' and disease' characteristics and disease activity parameters were collected at baseline. Based on the starting date for the first BT, patients were classified in two groups: i) before 2013 and ii) during or after 2013, since the nr-axSpA approval-date for TNFi in the country was July 2012. Gender distribution and other characteristics were compared between both groups using Chi-square and Student-t tests.

Results: In total, 385 axSpA patients initiated BT. Out of these, 266 initiated BT in period i) and 119 in period ii). The characteristics of patients in both groups are depicted in table 1. Importantly, no differences between period i) and ii) were observed regarding gender distribution ( $38 \%$ and $39 \%$ of women; $p=0.8$, respectively). Additionally, during period ii), the percentage of patients with $\mathrm{nr}-\mathrm{axSpA}$ was similar for both genders and out of all patients with $\mathrm{nr}-\mathrm{axSpA}$, the majority $(60 \%)$ were men. Overall, disease duration was shorter in period ii for both genders. Women in period ii) had significantly higher ASDAS, BASMI and CRP than women in period i) and higher ASDAS, BASDAI and BASFI than men in period ii).
Abstract OP0322 - Table 1 Patients characteristics stratified for starting first TNFi period and gender

\begin{tabular}{|c|c|c|c|c|c|c|}
\hline & \multicolumn{3}{|c|}{$2000-2012$} & \multicolumn{3}{|c|}{ 2013-2017 } \\
\hline & $\begin{array}{c}\text { Total } \\
n=266\end{array}$ & $\begin{array}{l}\text { Males } \\
n=164\end{array}$ & $\begin{array}{c}\text { Females } \\
n=102\end{array}$ & $\begin{array}{c}\text { Total } \\
n=119\end{array}$ & $\begin{array}{c}\text { Males } \\
n=72\end{array}$ & $\begin{array}{c}\text { Females } \\
\mathrm{n}=47\end{array}$ \\
\hline Age, yrs mean (SD) & $43.7(11.9)$ & $43.7(11.4)$ & $43.6(12.8)$ & $45.1(14.1)$ & $43.9(13.8)$ & $47.0(14.5)$ \\
\hline Smokers $\%$ & 44 & 47 & 39 & 49 & 47 & 51 \\
\hline $\begin{array}{l}\text { Disease duration, } \\
\text { months median (IQR) }\end{array}$ & $61.0(125.0)$ & $63.5(136.5)$ & $55.5(118.0)$ & $51.0(119.0)$ & $62.5(139.5)$ & $49.0(106.0)$ \\
\hline HLA-B27\% & 60 & 65 & 54 & 57 & 57 & 57 \\
\hline BASDAI median (IOR) & $6.0(2.3)$ & $5.7(2.5)$ & $6.9(2.4)$ & $6.0(2.4)$ & $5.8(2.2)^{+}$ & $6.5(2.6)^{+}$ \\
\hline ASDAS mean (SD) & $3.3(0.9)$ & $3.3(1.0)$ & $3.3(0.9)$ & $3.4(1.0)$ & $3.2(1.0)^{+}$ & $3.6(3.4)^{+}$ \\
\hline BASFI median (IQR) & $5.0(3.8)$ & $4.9(3.7)$ & $5.3(3.0)$ & $4.7(3.8)$ & $4.3(4.1)^{+}$ & $5.7(2.9)^{+}$ \\
\hline BASMI median (IIQR) & $2.1(2.2)$ & $2.4(2.4)$ & $2.0(1.4)^{*}$ & $2.4(2.0)$ & $2.2(2.2)$ & $2.7(1.7)^{*}$ \\
\hline CRP median (IQR) & $4.1(10.1)$ & $4.3(6.8)$ & $3.2(7.4)^{*}$ & $7.5(16.7)$ & $6.8(17.0)$ & $10.2(21.9)^{*}$ \\
\hline PtGA median (IQR) & $69(30.0)$ & $67(30.0)$ & $70(30.0)$ & $70(30.0)$ & $70(30.0)$ & $70(30.0)$ \\
\hline PGA median (IQR) & $45(40.0)$ & $40(40.0)$ & $50(40.0)$ & $45(30.0)$ & $40(30.0)$ & $45(30.0)$ \\
\hline $\begin{array}{l}\text { Peripheral } \\
\text { involvement, \% }\end{array}$ & 43 & 35 & 57 & 48 & 46 & 51 \\
\hline DMARDs, $\%$ & 49 & 47 & 51 & 51 & 46 & 60 \\
\hline $\mathrm{Nr}$-axSpA, $\%$ & - & - & - & 51 & 50 & 53 \\
\hline
\end{tabular}

Conclusions: In clinical practice, the frequency of women initiating BT have not increased since its approval for $\mathrm{nr}-\mathrm{axSpA}$. Additionally, women treated nowadays with BT have more objective parameters of disease activity than they used to do. This supports that when treating axSpA women (including nr-axSpA) with $\mathrm{BT}$, we are currently treating axSpA -and not fibromyalgia- patients.

Disclosure of Interest: None declared

DOI: 10.1136/annrheumdis-2018-eular.4190

\begin{tabular}{|l}
\hline OP0323 \\
ARE GENDER-SPECIFIC APPROACHES NEEDED IN \\
DIAGNOSING EARLY AXIAL SPONDYLOARTHRITIS? \\
DATA FROM THE SPONDYLOARTHRITIS CAUGHT \\
EARLY COHORT
\end{tabular}

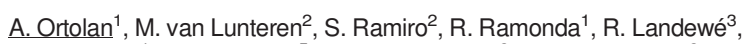
H. Dagfinrud ${ }^{4}$, L. Jacobsson ${ }^{5}$, D. van der Heijde ${ }^{2}$, F.A. van Gaalen².

${ }^{1}$ Rheumatology Unit, Department of Medicine DIMED, University of Padova, Padova, Italy; ${ }^{2}$ Department of Rheumatology, Leiden University Medical Center, Leiden; ${ }^{3}$ Department of Rheumatology, Amsterdam Rheumatology and Immunology Center, Amsterdam, Netherlands; ${ }^{4}$ Department of Rheumatology, Diakonhjemmet Hospital, Oslo, Nonway; ${ }^{5}$ Department of Rheumatology, University of Gothenburg, Gothenburg, Sweden

Background: Although gender differences have been observed in the severity of axial spondyloarthritis (axSpA), gender differences in disease presentation of early axSpA have not been thoroughly investigated.

Objectives: Our aim was to assess if the disease presents differently in males and females, and to evaluate if this has an impact on the diagnostic process.

Methods: Baseline data from the SPondyloArthritis Caught Early cohort, which includes patients with chronic back pain (CBP; $\geq 3$ months,$\leq 2$ years, onset $<45$ years), were analysed. Patients underwent a full diagnostic workup, including MRI and radiograph of the sacroiliac joints (MRI-SIJ and X-SIJ), to establish a diagnosis of axSpA. Characteristics of male and female patients with a definite diagnosis of axSpA (based on a level of confidence about the diagnosis $\geq 7$, as expressed by the physician on a $0-10$ rating scale) were compared. Regression models were built for 1) the whole CBP cohort stratified by gender to study which SpA features were associated most with diagnosis in each gender, and 2) for axSpA patients to test if gender was associated with imaging positivity (MRI SIJ+and/or $\mathrm{X}-\mathrm{SIJ}+$ ).

Results: Of the 719 CBP patients, 275 were male. With 146/275 (53.1\%) males and $155 / 444$ (34.9\%) females diagnosed as axSpA, males were more likely to be diagnosed with axSpA (OR 2.1,95\% Cl: 1.5 to 2.9). Despite similar symptom duration, male axSpA patients were younger at diagnosis (27.4 \pm 7.5 vs $29.5 \pm 7.8$ years; $p=0.021$ ). Presence of $S p A$ features was similar in male and female axSpA patients (table 1) except for HLA-B27 and imaging positivity, which were more common in male axSpA patients (HLA-B27 $+80 \%$ vs $60 \%$; $p<0.0001$ and positive imaging $78 \%$ vs $64 \% ; p=0.007$ ). Nevertheless, both these SpA features were still more prevalent in female axSpA patients than in non-axSpA patients, either females (HLA-B27 $+23 \%$ and imaging $7 \%$ ) or males (HLA-B27 $+34 \%$ and positive imaging $11 \%$ ) (all $\mathrm{p}<0.001)$. Moreover, in multivariable models with diagnosis as 